\title{
Further Analysis of Propagation of Plasma Waves in a "Spoke-Wheel" Magnetic Field
}

\author{
Richard R. Gold* \\ Aerodynamics and Propulsion Research Laboratory, Aerospace Corporation, El Segundo, Calif.
}

(Received July 22, 1964; revised March 24, 1965)

\begin{abstract}
A recent study by R. L. Liboff of electromagnetic wave propagation in plasmas with a specific type of two-dimensional applied magnetic field is continued in this paper. The plasma is assumed to be of uniform density in general, and a "spoke-wheel" magnetic field is considered which varies as the inverse radius. Perturbation series are obtained for the first Fourier component of the electric field for several limiting cases. Both the separate cases of a radial and a circular magnetic field are considered in the present work, the former in greater detail, and collisions are included. By suitable nondimensionalization, two parameters arise naturally in the present analysis, thus affording a more general solution which should be particularly useful in subsequent applications.
\end{abstract}

\section{Introduction}

In the present paper we shall extend the investigation initiated by Liboff [1964] of the cold plasma waves that may propagate in a specific type of two-dimensional magnetic field. A background discussion of this problem is given by Liboff. Both the separate cases of a radial and a circular magnetic field are considered in the present extension, the former in greater detail, and collisions are included. The four physical variables of the problem (electron density, collision frequency, magnetic field strength, and geometry) are included in two parameters which arise in the present analysis, in which perturbation series are generated for the electric field. The limiting cases are suggested naturally on this basis, and quadratures are obtained for the first-order terms in the perturbation parameter. The general form of the parameters results in a systematic solution which should be useful in subsequent applications.

\section{Analysis}

\subsection{Equations}

A collision term has been added to the matrix $\mathbf{A}$ in Liboff's formulation, and the governing equations were nondimensionalized in a convenient form to facilitate perturbation solutions (reference can be made to the detailed presentation [Gold, 1964] for specific intermediate equations and results). Assuming solutions of the form

$$
E_{\alpha}(\rho, \theta)=\sum_{\beta=0}^{\infty} E_{\alpha \beta}(\rho) e^{ \pm i \beta \theta}(\alpha=\rho, \theta, z),
$$

we obtain the following generalized governing equations for the electric field $\mathbf{E}$ :

$$
\begin{aligned}
\left( \pm i \beta / \rho^{2}\right)\left[\rho E_{\theta \beta}^{\prime}+E_{\theta \beta}-( \pm i \beta) E_{r \beta}\right] & =\left(n_{0} r_{0}\right)^{2}\left\{\left[1-A\left(U^{2}-Y_{r}^{2}\right)\right] E_{r \beta}+A Y_{r} Y_{\theta} E_{\theta \beta}-i A U Y_{\theta} E_{z \beta}\right\} \\
E_{\theta \beta}^{\prime \prime}+\frac{1}{\rho} E_{\theta \beta}^{\prime}-\frac{1}{\rho^{2}} E_{\theta \beta}-( \pm i \beta / \rho) E_{r \beta}^{\prime}+\left( \pm i \beta / \rho^{2}\right) E_{r \beta} & =-\left(n_{0} r_{0}\right)^{2}\left\{A Y_{r} Y_{\theta} E_{r \beta}+\left[1-A\left(U^{2}-Y_{\theta}^{2}\right)\right] E_{\theta \beta}+i A U Y_{r} E_{z \beta}\right\} \\
E_{z \beta}^{\prime \prime}+\frac{1}{\rho} E_{z \beta}^{\prime}-\frac{\beta^{2}}{\rho^{2}} E_{z \beta} & =-\left(n_{0} r_{0}\right)^{2}\left[i A U Y_{\theta} E_{r \beta}-i A U Y_{r} E_{\theta \beta}+\left(1-A U^{2}\right) E_{z \beta}\right],
\end{aligned}
$$

*Present position: Manager, Aerospace Physics Department, Space Systems Division, Hughes Aircraft Co., El Segundo, Calif. 
where the radial dimension has been nondimensionalized by some reference length $r_{0}$, i.e., $\rho=r / r_{0}$, and the prime denotes a derivative with respect to $\rho$. The following notations are employed: $A=X / U\left(U^{2}-Y^{2}\right), \quad Y^{2}=Y_{r}^{2}+Y_{\theta}^{2}, \quad U=1-i \omega_{c} / \omega, X=N e^{2} / \epsilon_{0} m \omega^{2}$, and $\mathbf{Y}=e \mathbf{B}_{0} / m \omega$, where $\omega$ is the angular wave frequency, $\omega_{c}$ the collision frequency, $n_{0}=2 \pi / \lambda_{0}$ the free space wave propagation constant, $\lambda_{0}$ the free space wavelength, $\epsilon_{0}$ the free space dielectric constant, and $N, e$, and $m$ are the number density, charge, and mass of the electron, respectively. We shall consider only the zeroth mode in the present analysis, and delete the subscript $\beta=0$ for convenience. The governing equations for the two explicit cases of interest; namely, case 1: $Y_{\theta}=0$, and case 2: $Y_{r}=0$, follow immediately from equations (2).

\subsection{Solution of Case 1}

The $E_{r}$ field is completely uncoupled from $E_{\theta, z}$ in this case and is nontrivial only for $\omega_{c}=0$, $X=1$, i.e., when the signal frequency is equal to the plasma frequency. The remaining two coupled equations define $E_{\theta}$ and $E_{z}$. Introducing $Y=a / \rho$, these become,

$$
L_{1} E_{\theta}=\frac{M \rho\left(\rho E_{\theta}-i N E_{z}\right)}{\rho^{2}-N^{2}}, \quad L_{0} E_{z}=\frac{M \rho\left(\rho E_{z}+i N E_{\theta}\right)}{\rho^{2}-N^{2}}
$$

where,

$$
\begin{aligned}
& L_{n} \equiv \frac{d^{2}}{d \rho^{2}}+\frac{1}{\rho} \frac{d}{d \rho}+\left[\left(n_{0} r_{0}\right)^{2}-\frac{n}{\rho^{2}}\right], \\
& M=\left(n_{0} r_{0}\right)^{2} X / U, \quad N=a / U .
\end{aligned}
$$

The four nondimensional constants of the problem, namely, $X, \omega_{c} / \omega, r_{0} / \lambda_{0}$, and $a$ combine to give two parameters $M$ and $N$ which must be ordered in the present expansion solution of (3).

CASE la: $|N| \gg 1$, i.e., $|a| \gg|U|=\left[1+\left(\omega_{c} / \omega\right)^{2}\right]^{1 / 2}$.

Let us assume that the plasma region is annular, $r_{1} \leqslant r \leqslant r_{2}$, and choose the reference length $r_{0}=r_{2}$ so that $r_{1} / r_{2} \leqslant \rho \leqslant 1$. The function $\left(\rho^{2}-N^{2}\right)^{-1}$ can then be expanded in inverse powers of $N$, and solutions for the zero mode components obtained in the form

$$
\mathbf{E}(\rho)=\sum_{n=0}^{\infty} \mathbf{E}^{(n)}(\rho) N^{-n}
$$

It is now necessary to order $M$ relative to $N$ systematically.

(i) $|M| \geqslant 0\left(\left|N^{2}\right|\right)$.

The solution for $\mathbf{E}^{(n)}$ degenerates into a null series.

(ii) $|\boldsymbol{M}|=0(|N|)$.

Since $|M / N|$ is of order one, the zeroth order equations remain coupled. In particular, to $0\left(N^{-1}\right)$ :

$$
\begin{array}{cc}
L_{1} E_{\theta}^{(0)}-\frac{i M \rho}{N} E_{z}^{(0)}=0, & L_{0} E_{z}^{(0)}+\frac{i M \rho}{N} E_{\theta}^{(0)}=0 \\
L_{1} E_{\theta}^{(1)}-\frac{i M \rho}{N} E_{z}^{(1)}=-\frac{M \rho^{2}}{N} E_{\theta}^{(0)}, & L_{0} E_{z}^{(1)}+\frac{i M \rho}{N} E_{\theta}^{(1)}=-\frac{M \rho^{2}}{N} E_{z}^{(0)}
\end{array}
$$

A single fourth-order linear equation can be obtained from (5) and, for example, a power series solution derived. Alternatively, power series solutions can be obtained directly from the system 
(5) and (6). Since the procedure is substantially different than the method of solution used in each of the remaining cases, we will not carry out the details at this time.

(iii) $|M|=0(1)$.

The zeroth and first-order equations are

$$
\begin{gathered}
B_{1} E_{\theta}^{(0)}(\xi)=0, \quad B_{0} E_{z}^{(0)}(\xi)=0 \\
B_{1} E_{\theta}^{(1)}=\frac{i M \xi}{\left(n_{0} r_{0}\right)^{3}} E_{z}^{(0)}, \quad B_{0} E_{z}^{(1)}=-\frac{i M \xi}{\left(n_{0} r_{0}\right)^{3}} E_{\theta}^{(0)},
\end{gathered}
$$

where the change of independent variable, $\xi=n_{0} n_{0} \rho$, introduces the Bessel operator of order $n$ and argument $\xi$,

$$
B_{n} \equiv \frac{d^{2}}{d \xi^{2}}+\frac{1}{\xi} \frac{d}{d \xi}+\left(1-\frac{n}{\xi^{2}}\right)
$$

Hence,

$$
\begin{aligned}
& E_{z}^{(0)}=c_{1} J_{0}(\xi)+c_{2} Y_{0}(\xi) \equiv P_{0}(\xi) \\
& E_{\theta}^{(0)}=c_{3} J_{1}(\xi)+c_{4} Y_{1}(\xi) \equiv P_{1}(\xi)
\end{aligned}
$$

The solution of (8) and (10) may be obtained using a procedure similar to the one used by Liboff [1964]. The particular solutions for $\mathbf{E}^{(1)}$ are derived by means of the substitutions, $E_{z}^{(1)}(\xi)=\epsilon_{z} \bar{P}_{0}$, $E_{\theta}^{(1)}(\xi)=\epsilon_{\theta} \bar{P}_{1}$, where $\bar{P}_{0}=c_{3} J_{0}+c_{4} Y_{0}$ and $\bar{P}_{1}=c_{1} J_{1}+c_{2} Y_{1}$. Thus,

$$
\begin{gathered}
\epsilon_{z}=\frac{-i M}{2\left(n_{0} r_{0}\right)^{3}} \int \frac{\xi P_{1}^{2}}{\bar{P}_{0}^{2}} d \xi+c_{5} \int \frac{d \xi}{\xi \bar{P}_{0}^{2}}+c_{6}, \\
\epsilon_{\theta}=\frac{i M \xi^{2}}{4\left(n_{0} r_{0}\right)^{3}}+c_{7} \int \frac{d \xi}{\xi \bar{P}_{1}^{2}}+c_{8} .
\end{gathered}
$$

The solutions for the corresponding homogeneous equations can be absorbed into the zeroth order solutions, and one obtains, to order $N^{-1}$,

$$
E_{z}=P_{0}+N^{-1} \bar{P}_{0} \epsilon_{z}, \quad E_{\theta}=P_{1}+N^{-1} \bar{P}_{1} \epsilon_{\theta},
$$

where $\vec{\epsilon}$ is given by (11). Note that in order to satisfy boundary conditions in general two arbitrary constants are required in both the zeroth and first-order solutions; the constants for the latter are included in the expressions for $\vec{\epsilon}$.

The extension of this solution to include $|M| \leqslant 0\left(\left|N^{-1}\right|\right)$ can be readily deduced from the preceding results.

CASE 1b: $|N| \ll 1, \quad$ i.e., $\quad|a| \ll|U|=\left[1+\left(\omega_{c} / \omega\right)^{2}\right]^{1 / 2}$.

Let $r_{0}=r_{1}$ in this case so that $1 \leqslant \rho \leqslant r_{2} / r_{1}$. The function $\left(\rho^{2}-N^{2}\right)^{-1}$ can now be expanded in ascending powers of $N$, and solutions for the zero mode components obtained in the form

$$
\mathbf{E}(\rho)=\sum_{n=0}^{\infty} \mathbf{E}^{(n)}(\rho) N^{n}
$$

It is necessary, once again, to order $M$ relative to $N$. 
(i) $|M| \geqslant 0\left(\left|N^{-1}\right|\right)$.

The solution for $\mathbf{E}^{(n)}$ degenerates into a null series.

(ii) $|M|=0(1)$.

$$
\begin{gathered}
B_{1} E_{\theta}^{(0)}(\zeta)=0, \quad B_{0} E_{z}^{(0)}(\zeta)=0 \\
B_{1} E_{\theta}^{(1)}=\frac{-i M}{n_{0} n_{0} \alpha \zeta} E_{z}^{(0)}, \quad B_{0} E_{z}^{(1)}=\frac{i M}{n_{0} n_{0} \alpha \zeta} E_{\theta}^{(0)},
\end{gathered}
$$

where, $\alpha=(1-X / U)^{1 / 2}$ is the index of refraction with no magnetic field, and the Bessel operator involves the argument $\zeta=n_{0} r_{0} \alpha \rho$. Proceeding as before, one obtains, to order $N$ :

$$
\begin{gathered}
E_{z}(\zeta)=P_{0}+N \bar{P}_{0} \epsilon_{z}, \quad E_{\theta}(\zeta)=P_{1}+N \bar{P}_{1} \epsilon_{\theta}, \\
\epsilon_{z}=\frac{-i M}{2 n_{0} r_{0} \alpha} \ln \zeta+c_{5} \int \frac{d \zeta}{\zeta \bar{P}_{0}^{2}}+c_{6}, \\
\epsilon_{\theta}=\frac{i M}{2 n_{0} r_{0} \alpha} \int \frac{P_{0}^{2}}{\zeta \bar{P}_{1}^{2}} d \zeta+c_{7} \int \frac{d \zeta}{\zeta \bar{P}_{1}^{2}}+c_{8} .
\end{gathered}
$$

(iii) $|M|=0(|N|)$.

$$
\begin{gathered}
B_{1} E_{\theta}^{(0)}(\xi)=0, \quad B_{0} E_{z}^{(0)}(\xi)=0 ; \\
B_{1} E_{\theta}^{(1)}=\frac{M}{N\left(n_{0} r_{0}\right)^{2}} E_{\theta}^{(0)}, \quad B_{0} E_{z}^{(1)}=\frac{M}{N\left(n_{0} r_{0}\right)^{2}} E_{z}^{(0)} .
\end{gathered}
$$

The particular solutions of (18) can now be assumed to be of the form, $E_{z, \theta}^{(1)}=\epsilon_{z, \theta} E_{z, \theta}^{(0)}$, and one obtains:

$$
\mathbf{E}(\xi)=\mathbf{E}^{(0)}(1+N \vec{\epsilon})
$$

where $E_{z}^{(0)}=P_{0}(\xi), E_{\theta}^{(0)}=P_{1}(\xi)$,

and

$$
\begin{aligned}
\epsilon_{z} & =\frac{M}{N\left(n_{0} r_{0}\right)^{2}} \int \frac{d \xi}{\xi P_{0}^{2}} \int \xi P_{0}^{2} d \xi+c_{5} \int \frac{d \xi}{\xi P_{0}^{2}}+c_{6}, \\
\epsilon_{\theta} & =\frac{M}{N\left(n_{0} r_{0}\right)^{2}} \int \frac{d \xi}{\xi P_{1}^{2}} \int \xi P_{1}^{2} d \xi+c_{7} \int \frac{d \xi}{\xi P_{1}^{2}}+c_{8} .
\end{aligned}
$$

It should be pointed out that a variable electron density could be included in the present quadratures, for example (or in the preceding solutions by similar manipulations), in the following manner. Let $\chi(\xi)=X(\xi) / X_{m}$ be the normalized radially varying electron density, where $X_{m}$ is the maximum value of $X$ in the plasma (hence, $\chi \leqslant 1$ ). A new constant parameter $M_{1}$ can then be defined in place of $M$, namely, $M=M_{1} \chi$, where $M_{1}=\left(n_{0} r_{0}\right)^{2} X_{m} / U$. The above solution for $\epsilon_{z}$ would then become:

$$
\epsilon_{z}=\frac{M_{1}}{N\left(n_{0} r_{0}\right)^{2}} \int \frac{d \xi}{\xi P_{0}^{2}} \int \xi \chi P_{0}^{2} d \xi+c_{5} \int \frac{d \xi}{\xi P_{0}^{2}}+c_{6}
$$

The extension of case lb to include $|M| \leqslant 0\left(\left|N^{2}\right|\right)$ can be readily deduced from the preceding results. 
CASE lc: $|N|=0(1)$, i.e., $|a|=0(|U|)=0\left(\sqrt{1+\left(\omega_{c} / \omega\right)^{2}}\right)$.

Since $\left(\rho^{2}-N^{2}\right)^{-1}$ can no longer be expanded, we are left with the single parameter $M$ in (3).

(i) $|M| \gg 1$.

An expansion in inverse powers of $M$ would result in a degenerate null series for the electric field.

(ii) $|M|=0(1)$.

No parameter is available for obtaining expansion solutions to (3). It should be remarked, however, that only $|M|$ and $|N|$ were specified in the discussion of cases la and $1 \mathrm{~b}$. Thus the quantities $|a / U|$ and $\left|\left(n_{0} r_{0}\right)^{2} X / U\right|$ or $\left|\left(n_{0} r_{0}\right)^{2} X / a\right|$ were specified. However, the actual magnitudes of $n_{0} r_{0}, a, X$, and $|U|$ were restricted only insofar as they affect these ratios. By further specifying these parameters, the solutions in cases la and lb can be simplified, say, in evaluating the quadratures. Limited solutions can also be obtained in the present case on the same basis. For example, let us now nondimensionalize the radial dimension by the free space wave propagation constant so that $x=n_{0} r$. In place of (3), one obtains the following system of equations:

$$
B_{1} E_{\theta}=\frac{\bar{M} x\left(x E_{\theta}-i \bar{N} E_{z}\right)}{x^{2}-\bar{N}^{2}}, \quad B_{0} E_{z}=\frac{\bar{M} x\left(x E_{z}+i \bar{N} E_{\theta}\right)}{x^{2}-\bar{N}^{2}}
$$

where $Y=\bar{a} / x$,

and

$$
\bar{M}=X / U, \quad \bar{N}=\bar{a} / U .
$$

Hence, by restricting the analysis to sufficiently large value of $\lambda_{0}$ (for fixed $r_{1}$ and $r_{2}$ ), so that $x<<1$, expansion solutions in powers of $x$ could be generated. Similarly, for sufficiently small value of $\lambda_{0}, x>>1$, and expansion solutions in inverse powers of $x$ could be obtained.

$$
\text { (iii) }|\boldsymbol{M}| \ll 1 \text {, i.e., }\left(n_{0} r_{0}\right)^{2} X \ll\left[1+\left(\omega_{c} / \omega\right)^{2}\right]^{1 / 2} \text {. }
$$

Assuming solutions in the form

$$
\mathbf{E}(\rho)=\sum_{n=0}^{\infty} \mathbf{E}^{(n)}(\rho) M^{n}
$$

eq $(3)$ reduce to

$$
\begin{aligned}
& L_{1}\left\{\sum_{n} E_{\theta}^{(n)} M^{n}\right\}=\frac{M \rho}{\rho^{2}-N^{2}} \sum_{n}\left(\rho E_{\theta}^{(n)}-i N E_{z}^{(n)}\right) M^{n}, \\
& L_{0}\left\{\sum_{n} E_{z}^{(n)} M^{n}\right\}=\frac{M \rho}{\rho^{2}-N^{2}} \sum_{n}\left(\rho E_{z}^{(n)}+i N E_{\theta}^{(n)}\right) M^{n},
\end{aligned}
$$

which can be solved, provided that $r_{1} / r_{2} \leqslant \rho \leqslant 1$, i.e., by selecting $r_{0}=r_{2}$ for the reference length. General recursion relations can be obtained in this case $(n=0,1,2,3, \ldots)$ :

$$
\begin{aligned}
& L_{1} E_{\theta}^{(0)}=0, \quad L_{0} E_{z}^{(0)}=0, \\
& L_{1} E_{\theta}^{(n+1)}=\frac{\rho\left(\rho E_{\theta}^{(n)}-i N E_{z}^{(n)}\right)}{\rho^{2}-N^{2}}, \quad L_{0} E_{z}^{(n+1)}=\frac{\rho\left(\rho E_{z}^{(n)}+i N E_{\theta}^{(n)}\right)}{\rho^{2}-N^{2}} .
\end{aligned}
$$

To order $M$, therefore,

$$
\mathbf{E}(\xi)=\mathbf{E}^{(0)}(1+M \vec{\epsilon}),
$$


where

$$
E_{z}^{(0)}=P_{0}(\xi), \quad E_{\theta}^{(0)}=P_{1}(\xi), \quad \xi=n_{0} r_{0} \rho
$$

and

$$
\begin{aligned}
& \boldsymbol{\epsilon}_{z}=\frac{1}{\left(n_{0} r_{0}\right)^{2}} \int \frac{d \xi}{\xi P_{0}^{2}} \int \frac{\xi^{2} P_{0}\left(\xi P_{0}+i n_{0} r_{0} N P_{1}\right)}{\xi^{2}-\left(n_{0} r_{0} N\right)^{2}} d \xi+c_{5} \int \frac{d \xi}{\xi P_{0}^{2}}+c_{6}, \\
& \epsilon_{\theta}=\frac{1}{\left(n_{0} r_{0}\right)^{2}} \int \frac{d \xi}{\xi P_{1}^{2}} \int \frac{\xi^{2} P_{1}\left(\xi P_{1}-i n_{0} r_{0} N P_{0}\right)}{\xi^{2}-\left(n_{0} r_{0} N\right)^{2}} d \xi+c_{7} \int \frac{d \xi}{\xi P_{1}^{2}}+c_{8} .
\end{aligned}
$$

A variable electron density could be included in the manner outlined at the end of the discussion of case $1 b$ (iii).

\subsection{Solution of Case 2}

In this case $E_{\theta}$ is completely uncoupled from $E_{r}$ and $E_{z}$ and is independent of the applied magnetic field $B_{\theta}$. Indeed, the following exact solution is immediately apparent:

$$
E_{\theta}=c_{1} J_{1}(\zeta)+c_{2} Y_{1}(\zeta) \equiv P_{1}(\zeta)
$$

where $\zeta=n_{0} r_{0} \rho(1-X / U)^{1 / 2}$. Since the reduced eq (2a) is algebraic, the equations for $E_{r}$ and $E_{z}$ can be uncoupled. Assuming $Y=\frac{b}{\rho}$, one obtains

$$
\begin{gathered}
E_{r}=\frac{(i X / \alpha U) \tilde{N} \rho}{\rho^{2}-\tilde{N}^{2}} E_{z}, \quad L_{0} E_{z}(\rho)=\frac{M \rho^{2}}{\rho^{2}-\tilde{N}^{2}} E_{z}, \\
M=\left(n_{0} r_{0}\right)^{2} X / U, \quad \tilde{N}=\frac{b}{\alpha U}, \quad \alpha=\left(1-\frac{X}{U}\right)^{1 / 2} .
\end{gathered}
$$

The problem reduces to the solution of (30b) for $E_{z}$ in terms of the two explicit parameters, $M$ and $\tilde{N}$. $\quad E_{r}$ can then be obtained immediately from (30a). An approach similar to the one employed in case 1 can be adopted in order to solve $(30 \mathrm{~b})$, although this is not the only possible procedure. We will limit our analysis of this problem to the following brief consideration.

The right-hand side of (30b) can be neglected if $\left|M \rho^{2} / \rho^{2}-\tilde{N}^{2}\right| \ll 1$. This condition is satisfied if $|M| \ll 1, r_{0}$ is selected such that $\rho \leqslant 1$ and the possibility of $\tilde{N}$ being real with $\tilde{N} \approx \rho$ is excluded. In this case $(30 \mathrm{~b})$ becomes:

$$
B_{0} E_{z}(\xi)=\frac{M_{1} \xi^{2} \chi E_{z}}{\left(n_{0} r_{0}\right)^{2}\left(\xi^{2}-n_{0}^{2} r_{0}^{2} \tilde{N}^{2}\right)}
$$

and a series solution in powers of $M_{1}$ can be obtained. A variable electron density has been included as outlined at the end of the discussion of case $1 \mathrm{~b}$ (iii). It follows that:

$$
\begin{gathered}
E_{z}(\xi)=E_{z}^{(0)}\left(1+M_{1} \epsilon_{z}\right), \quad E_{z}^{(0)}=P_{0}(\xi), \\
\epsilon_{z}=\frac{1}{\left(n_{0} r_{0}\right)^{2}} \int \frac{d \xi}{\xi P_{0}^{2}} \int \frac{\xi^{3} \chi P_{0}^{2}}{\xi^{2}-\left(n_{0} r_{0} \tilde{N}\right)^{2}} d \xi+c_{3} \int \frac{d \xi}{\xi P_{0}^{2}}+c_{4} .
\end{gathered}
$$

The corresponding solution for $E_{r}$ is given by (30a). 


\section{Conclusions}

The introductory analysis has been completed on a class of electromagnetic waves which will propagate through a cold plasma in which is imbedded a specific type of cylindrical magnetic field. The plasma is assumed to be of uniform density and collision frequency, and a so-called "spoke-wheel" magnetic field is applied which is both anisotropic and inhomogeneous (varying as the inverse radius). Perturbation series were obtained for the first Fourier component of the electric field by systematically ordering the two parameters of the problem. The results are given in quadrature form and exhibit the correct limiting character in the lowest order expressions. Similar studies of guided electromagnetic waves through cylindrical plasmas with imbedded $B_{z}$ fields (constant) have been made [Liboff, 1964]. The electric field components are uncoupled in these cases, whereas in the present analysis a cylindrical magnetic field is applied and the electric field components are severely coupled.

It remains to fit these solutions to specific geometries. The most physically relevant of these is the class of annular geometries referred to in the text. The formulation and solution of a meaningful boundary value problem based on the present results would enable one to evaluate quantitatively the effect of a variable magnetic field on propagation as a function of the several physical parameters of the problem.

\section{References}

Gold, R. R. (1964), Further analysis of propagation of plasma waves in a "spoke-wheel" magnetic field, Aerospace Corp. Internal Memo.

Liboff, R. L. (1964), Propagation of plasma waves in a "spoke-wheel" magnetic field, J. Res. NBS 68D (Radio Sci.), No. 3, 325-331.

(Paper 69D9-556) 